\title{
Does reducing spasticity translate into functional benefit? An exploratory meta-analysis
}

\author{
H P Francis, D T Wade, L Turner-Stokes, R S Kingswell, C S Dott, E A Coxon
}

J Neurol Neurosurg Psychiatry 2004;75:1547-1551. doi: 10.1136/jnnp.2003.025551

See end of article for authors' affiliations ......................

Correspondence to: Dr H Francis, Ipsen Limited, 190 Bath Road, Slough, SL1 3XE, Berkshire, UK; hilary.francis@ipsen.com

Received 12 August 2003 In revised form 22 January 2004 Accepted 22 January 2004

\begin{abstract}
Background: Spasticity and loss of function in an affected arm are common after stroke. Although botulinum toxin is used to reduce spasticity, its functional benefits are less easily demonstrated. This paper reports an exploratory meta-analysis to investigate the relationship between reduced arm spasticity and improved arm function.

Method: Individual data from stroke patients in two randomised controlled trials of intra-muscular botulinum toxin were pooled. The Modified Ashworth Scale (elbow, wrist, fingers) was used to calculate a "Composite Spasticity Index". Data from the arm section of the Barthel Activities of Daily Living Index (dressing, grooming, and feeding) and three subjective measures (putting arm through sleeve, cleaning palm, cutting fingernails) were summed to give a "Composite Functional Index". Change scores and the time of maximum change were also calculated.

Results: Maximum changes in both composite measures occurred concurrently in 47 patients. In 26 patients the improvement in spasticity preceded the improvement in function with 18 showing the reverse. There was a definite relationship between the maximum change in spasticity and the maximum change in arm function, independent of treatment $(\rho=-0.2822, p=0.0008, n=137)$. There was a clear relationship between the changes in spasticity and in arm function in patients treated with botulinum toxin (Dysport) at 500 or 1000 units ( $\rho=-0.5679, p=0.0090, n=22 ; \rho=-0.4430, p=0.0018, n=47$ ), but not in those treated with placebo or 1500 units.

Conclusions: Using a targeted meta-analytic approach, it is possible to demonstrate that reducing spasticity in the arm is associated with a significant improvement in arm function.
\end{abstract}

$\mathrm{S}$ pasticity arises from upper motor neurone dysfunction in a number of neurological disorders, including stroke. The impact for individual patients varies enormously, ranging from relatively minor effects on quality of movement, to a more substantial contribution to the difficulties experienced by carers in helping with most activities of daily living (ADL).

Intra-muscular injection of botulinum toxin is increasingly used to treat focal spasticity. Controlled and uncontrolled studies $^{1-7}$ have reported statistically significant changes in spasticity (Modified Ashworth Scale, $\mathrm{MAS}^{8}$ ) following its use.

In these studies, and clinically, there are two underlying assumptions: first, that spasticity contributes to the limitation of activities, and, second, that reducing the spasticity will therefore bring about an improvement in function. A number of publications report improved attainment of pre-specified goals, ${ }^{9}$ overall benefit, ${ }^{2}$ and reduced carer burden associated with reduced spasticity. ${ }^{10}$ However, in the studies reported so far, the observed reductions in spasticity have rarely been associated with clear gains in functional activity.

There are several possible reasons for the failure to show functional gain for the patient in most studies of anti-spastic agents. ${ }^{11}$ The treatments may be ineffective in reducing spasticity, but this is unlikely since benefit has been demonstrated in a number of double-blind, placebo-controlled trials. ${ }^{1-4} 6^{12-14}$ It is possible that spasticity does not contribute to the limitation of function and that the underlying weakness is the only significant cause of activity limitation. ${ }^{15}{ }^{16}$ However, the most likely reason is that all studies have been inadequately powered to detect functional gain or that the measures used were insufficiently sensitive. ${ }^{9} 17$

Botulinum toxin is injected into individual muscles to reduce focal spasticity. Most standardised measures of disability, such as the Barthel Index, provide a global assessment of bodily function and are thus unlikely to detect changes occurring in one or two activities among a battery of tasks unaffected by the intervention. For example, a significant improvement in dressing from "requiring some help" to being "independent" would contribute only one point out of 20 to a Barthel score. ${ }^{18}$ Goal attainment scaling ${ }^{19} 20$ has been used in some studies to provide more targeted measurement, ${ }^{21}$ but presents theoretical problems in providing direct comparison between individual patients or groups. An alternative approach is to select a subset of functional measures from within a standard scale, which best assess function in the relevant part of the body.

Meta-analysis is increasingly used to extract common data sets from different studies and to provide further analysis of the combined study populations. However, it requires a degree of uniformity within the original study designs (selection, randomisation, measurement instruments, etc). Pooling of data may include summation of individual scores or data transformations where techniques have differed slightly between trials.

In this study we have used a meta-analytic approach to perform further analyses on pooled individual data from two trials of botulinum toxin for arm spasticity after stroke, ${ }^{12}$ in order to examine the relationship between changes in spasticity and changes in functional performance. A selected subset of functional measures was used to identify changes in arm function.

\section{METHODS}

The data from two randomised, double-blind, placebocontrolled trials of botulinum toxin were used. The studies

Abbreviations: ADL, activities of daily living; MAS, Modified Ashworth Scale 
Table 1 Summary of data from patients studied

\begin{tabular}{|c|c|c|c|c|c|}
\hline & Study $1^{1}$ & Study $2^{2}$ & Overall, baseline & Overall, week 4 & Overall, week 16 \\
\hline Number of patients & 83 & 59 & 142 & & \\
\hline \multicolumn{6}{|l|}{ Treatment } \\
\hline Placebo & 19 & 32 & 51 & & \\
\hline Dysport 500 units & 22 & & 22 & & \\
\hline Dysport 1000 units & 22 & 27 & 49 & & \\
\hline Dysport 1500 units & 19 & & 19 & & \\
\hline Men & & & 77 & & \\
\hline Age, mean (SD) years & & & $64.0(12.8)$ & & \\
\hline Barthel Index, median (range) & $13(3-20)$ & $13(1-20)$ & $13(1-20)$ & $13.5(1-20)$ & $13.5(1-20)$ \\
\hline Subset of Barthel Index (items 3, 5, and 8), median (range) & $3(1-5)$ & $3(1-5)$ & $3(1-5)$ & $3(1-5)$ & $3(1-5)$ \\
\hline Total Composite Spasticity Index, median (range) & $11(3-15)$ & $11(1-15)$ & $11(1-15)$ & $8(0-15)$ & $9(0-15)$ \\
\hline Total Composite Functional Index, median (range) & $7(1-15)$ & $7(1-15)$ & $7(1-15)$ & $8(1-15)$ & $8(1-17)$ \\
\hline
\end{tabular}

were chosen on the basis that their designs were sufficiently similar to allow pooling of individual data. They were sponsored by the same company (Ipsen), used the same botulinum toxin (Dysport), and included directly comparable measures recorded at the same time intervals. The first trial was a dose-ranging study, comparing the effects of three different doses of botulinum toxin (500, 1000, 1500 units Dysport $)^{1}$ against placebo. The second used a fixed dose (1000 units Dysport). ${ }^{2}$ All patients were assessed at baseline (week 0 ) and at weeks 4, 8, 12, and 16, which allowed the temporal relationship between changes in spasticity and function to be examined. All assessments were undertaken by investigators who were unaware of the patient's treatment, and trained in the use of the measures assessed

All patients had had a stroke at least 3 months prior to entry, and had significant spasticity in the affected arm (scoring greater than 2 of 4 on the $\mathrm{MAS}^{8}$ in at least two of three joints in the arm). Background information was collected on all patients on entry to the study and all patients gave informed consent. Patients who were receiving ongoing rehabilitation, primarily physiotherapy, continued with this treatment after botulinum toxin injection.

Data from the following measures were used in our analyses:

- The MAS for spasticity. ${ }^{8}$ As published this is scored 0-4, with a $1+$ grade, but for data analysis the scores were adjusted to give a $0-5$ score range ( $1+$ became 2,2 became 3 , and so on). This scale was applied to the affected flexors at the elbow, wrist, and fingers. The scores were added, giving a "Composite Spasticity Index" from 0 to 15.

- The scores of the study subjective assessments (cleaning palm, cutting fingernails, and putting arm through sleeve) were added to those for three items from the Barthel Index ${ }^{18}$ typically involving upper limb function (items 3, 5, and 8-grooming, feeding, and dressing) to form a "Composite Functional Index".

Since the scales for the different assessments were scored in opposite directions, the subjective scores were adjusted for analysis, with low scores reflecting more severe impairment and high scores representing little impairment (see Appendix A). This gave a range for the "Composite Functional Index" of $0-17$.

Two additional variables for each component (spasticity and function) were also calculated:

- A change score, comparing the index at each time point with the score at baseline.

- The peak time (assessment point), when the maximum change was first observed. If the peak occurred at only one point, that time was recorded, but if the peak response was observed at two or more points, the time at which it was first observed was recorded.

We investigated the timing of the maximum improvement in each index for each patient and the inter-relationship between the maximum change in spasticity index and the maximum change in functional index (that is, the relationship between the maximum change seen in each measure in each patient over the 16 weeks of observation). We also investigated the relationship between extent of change in spasticity and extent of change in arm function, regardless of the time of that change, using Spearman's ranked correlation coefficient.

\section{RESULTS}

Full details of the patients are given in the original papers, ${ }^{12}$ but important relevant information is summarised in table 1. A total of 142 patients were recruited to the two studies and on entry data were available from 141. Some data were not available for every patient at every time point, accounting for the reduction to 137 patients in some analyses.

The timing of the maximum improvement in Composite Spasticity Index and in Composite Functional Index in individual patients is shown in table 2 . Ten patients showed no improvement at any time point. In addition, 31 showed a reduction in spasticity with no change in function, while only five showed an improvement in function without a change in spasticity. Where changes in both measures were observed, the time of maximum change coincided in 47 patients, usually $(\mathrm{n}=34)$ at 4 weeks. Interestingly, 26 showed a maximum change in spasticity before a maximum change in arm function, while only 18 showed an improvement in arm function before reaching a maximum change in spasticity.

The relationship between the maximum change in spasticity and that in arm function in individual patients is shown in fig 1. Where available, data were included for each patient, independent of treatment. There was a clear association between improvement in spasticity and improvement in arm function $(\rho=-0.2822, p=0.0008, n=137)$.

Examination of the separate treatment groups (fig 2) showed that in those patients treated with placebo, there was only a weak association between the maximal change in spasticity and the maximal change in arm function, which was not statistically significant $(\rho=-0.2223, p=0.1775$, $\mathrm{n}=49$ ). The extent of the changes in these patients was smaller than in the individuals receiving the active treatment. In addition, 17 of these patients failed to show any improvement in spasticity and/or function.

In patients receiving botulinum toxin (Dysport) at 500 or 1000 units, the extent of the maximal changes in spasticity and in arm function were greater than in the placebo-treated group. There was a clear and statistically significant association 
Table 2 Timing of maximum improvement (first point) for spasticity and arm function, all treatments

\begin{tabular}{|c|c|c|c|c|c|c|}
\hline \multirow[b]{2}{*}{ Arm function } & \multirow[b]{2}{*}{ No improvement } & \multicolumn{4}{|c|}{ Spasticity } & \multirow[b]{2}{*}{ Total } \\
\hline & & Week 4 & Week 8 & Week 12 & Week 16 & \\
\hline No improvement & 10 & 21 & 6 & 3 & 1 & 41 \\
\hline Week 4 & 3 & 34 & 5 & 6 & 2 & 50 \\
\hline Week 8 & 0 & 6 & 10 & 1 & 1 & 18 \\
\hline Week 12 & 1 & 3 & 5 & 1 & 3 & 13 \\
\hline Week 16 & 1 & 6 & 3 & 3 & 2 & 15 \\
\hline Total & 15 & 70 & 29 & 14 & 9 & 137 \\
\hline
\end{tabular}

Underline, no improvement in one measure with improvement in the other; bold, concomitant maxima; below diagonal, spasticity improved before function; above diagonal, function improved before maximum change in spasticity.

between these values, $(\rho=-0.5679, \mathrm{p}=0.0090, \mathrm{n}=22$ and $\rho=-0.4430, \mathrm{p}=0.0018, \mathrm{n}=47$, respectively).

Patients treated with botulinum toxin (Dysport) 1500 units also showed marked changes in spasticity, compared with placebo. There was a weak association between the maximal changes in spasticity and function, but this was not statistically significant $(\rho=-0.1186, p=0.6393, n=19)$. In none of the patients was a deterioration in function observed and in the majority (14/19) function clearly improved.

\section{DISCUSSION}

This is the first study to investigate the relationship between changes in spasticity and changes in function in a large group of patients, and has the particular advantage of having detailed data from five time points collected in a standardised manner. The results show that reducing spasticity can lead to improved function. They suggest that a moderate dose of botulinum toxin reduces spasticity sufficiently to allow function to improve, without causing a substantial decrease in strength, which may further impede function.

This analysis has several strengths. The main analysis was undertaken without regard to the mechanism of spasticity reduction and the results show a correlation between the change in spasticity and the change in function independent of treatment. Data collection was of a high quality in a large number of patients.

In many patients treated with placebo, there were improvements in both spasticity and function. This is to be expected since they may still have been improving, spontaneously or in response to therapy, following their stroke. The lack of a statistically significant correlation between these

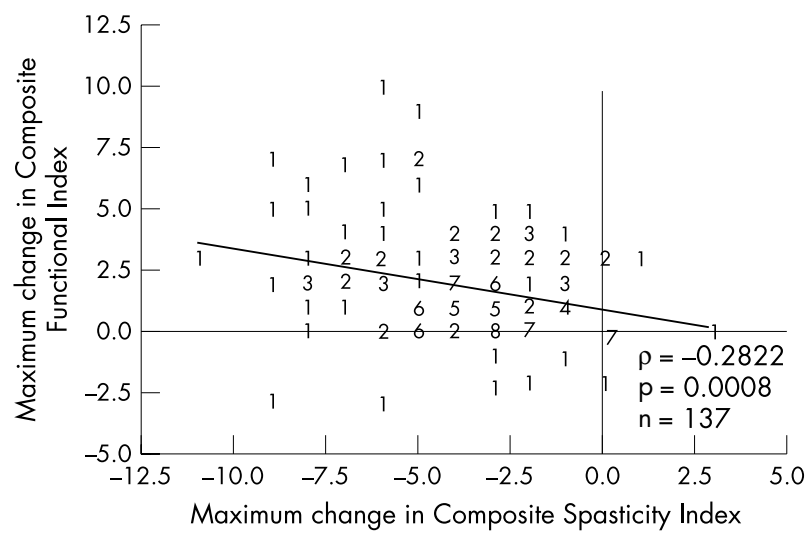

Figure 1 The relationship between the maximal change in Composite Functional Index and the maximal change in Composite Spasticity Index in 137 stroke patients followed over 16 weeks. The numeral at each point indicates the number of patients exhibiting that change. changes may simply be due to the relatively high proportion of patients who failed to show any change in spasticity and/or function $(n=17)$. However, it is possible that the reduction in spasticity seen after injecting saline (placebo) may be qualitatively different, which might account for the reduced strength of the relationship.

In patients treated with botulinum toxin, the changes in both spasticity and function were consistently greater than in those treated with placebo. The number of patients showing no change in spasticity and/or function was higher among those treated with placebo ( 23 of $49 ; 47 \%$ ) than among those receiving Dysport at any dose (27 of $88 ; 31 \%$ ).

In the small number of patients treated with the highest dose of botulinum toxin (Dysport 1500 units), there were marked changes in spasticity. In this group, however, the improvements in function tended not to be as large as in the other groups and there was no significant correlation between these measures. It is possible that this high dose caused an over-weakening of the injected muscles, further adding to the disability. Although it is also possible that the lack of a statistically significant association is simply a reflection of the low power relating to the smaller number of patients receiving this treatment.

In addition to the association between changes in spasticity and function, these analyses demonstrate a time lag between these changes in many patients. This delay is unsurprising because the patients are likely to need time to learn how to use any reduced muscle tone. Indeed this study supports the need for botulinum toxin use to be combined with active rehabilitation (as it was with many of these patients) so that they may capitalise on the window of opportunity offered by any reduction in spasticity.

Among the data from these patients, however, there were individuals in whom the maximal change in function preceded the maximal change in spasticity. Where this occurred it is presumed that benefit in function does not require a maximal change in spasticity and may peak whilst spasticity continues to improve.

An alternative explanation may lie in the relatively long gaps (4 weeks) between the assessments. For example it is possible that the peak changes may have occurred in both parameters at 6 weeks after treatment, but were recorded at 4 and 8 weeks, since no measurements were made at 6 weeks. This relatively infrequent assessment was one of the limitations of this study, reducing the accuracy of the time point of maximum change.

There were other limitations to this study. The measures used were relatively crude, making it more difficult to investigate the inter-relationship between spasticity and function. Nonetheless, the data were much more extensive than those in most other studies. A further criticism may be that the summation of individual scores into a single measure is not accepted in all circles as a valid technique, 
Combined data Placebo

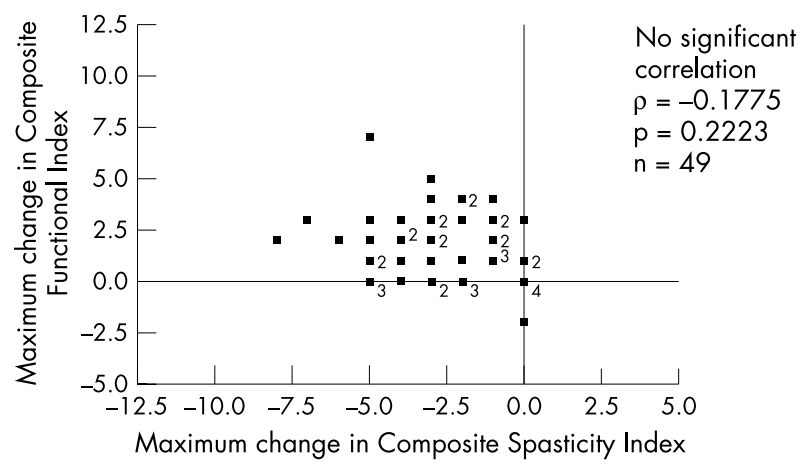

Combined data

Dysport 1000 units

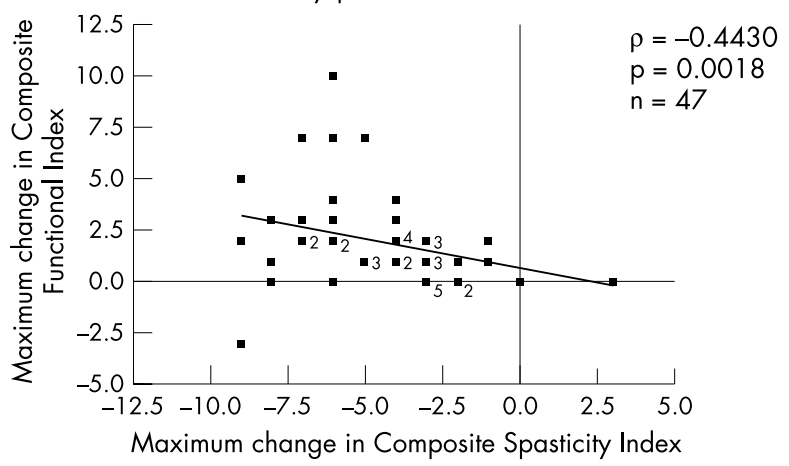

Study 1

Dysport 500 units

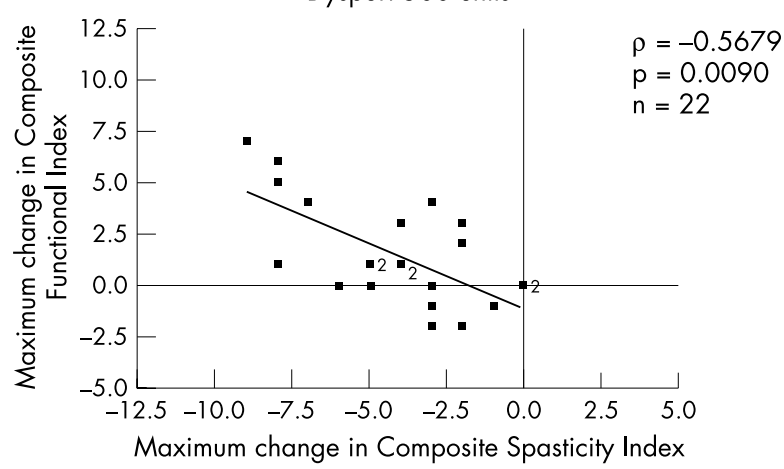

Study 1

Dysport 1500 units

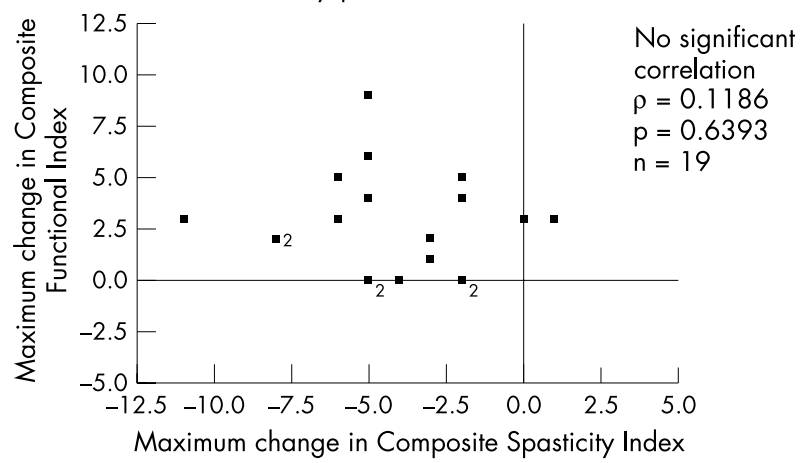

Figure 2 The relationship between the maximal change in Composite Functional Index and the maximal change in Composite Spasticity Index in different treatment groups in 137 stroke patients followed over 16 weeks. The numeral to the right of some data points indicates the number of patients exhibiting that change, if $>1$.

particularly where these include items from the different dimensions of functional ability. However, this approach has been quite widely applied in many areas of rehabilitation. Bakheit et $a l^{1}$ assessed spasticity on the whole arm by summing the MAS score at the different joints (elbow, wrist, and fingers) and Snow et al ${ }^{12}$ applied a summed score for the different dimensions of pain, deformity, and ease of care.

Another limitation in these studies was the inconsistent application of physiotherapy, which may have confounded some of the analyses. Throughout the course of the study, patients continued to receive their established regimen for this treatment. This varied from zero to 10 hours per week. There was a tendency for the patients receiving physiotherapy in addition to botulinum toxin, to gain greater benefit than those who received no physiotherapy and placebo treatment, but the large spread of these data precluded extensive analysis.

Previous studies may have failed to show functional benefit for several reasons. The amount of benefit is quantitatively (but not clinically) small and global measures of ADL will fail to detect it, as will small studies with inadequate power. Furthermore, in many patients, the timing of maximum change in spasticity occurs before the maximum change in function. If the primary outcome is only measured at one time point (as is traditionally required in randomised controlled trials) then the study risks missing at least one aspect of any benefit. Thirdly, many studies (especially those of oral drugs) do not include active rehabilitation and consequently patients may not realise the potential functional benefits of effective treatment.

From the patient's point of view two points need emphasis. First, relieving spasticity may often be a goal in its own right, just as relieving pain is a sufficient goal for analgesic drugs.
Spasticity is often uncomfortable, and sometimes very painful. Likewise simply making it easier to care for the spastic arm may be a valid, and even potentially costeffective, goal. Second, moving from requiring "some help" to needing "no help" in dressing for example, may only score one point on an ADL scale, but for the patient can make the difference between waiting for a carer in the morning and being able to get up at the time they choose.

The value of these small changes to the patients, despite a lack of statistical significance, was reflected in the single dose study, ${ }^{2}$ where $92 \%$ of patients reported a global benefit after botulinum toxin treatment, compared with $50 \%$ receiving placebo $(p=0.007$, logistic regression and odds ratio). The investigators' rating of the same item was $88 \%$ compared with $50 \%$, respectively $(\mathrm{p}=0.002$, logistic regression and odds ratio).

We would recommend that future studies take a number of factors into account. They should have at least two primary outcome measures to reflect the different aspects of ill health, ${ }^{22}$ one at the level of impairment (spasticity) and one at the level of activities (function). These two primary outcome measures should be measured at a number of different time points, ideally on a number of occasions and at short intervals. Where possible, the functional measure should be restricted to those activities which might be expected to show benefit. Studies should also consider using goal attainment scaling or a similar approach (for example, global assessment of benefit) to acknowledge the various goals of treating focal spasticity. ${ }^{20}$ Finally, patients should have access to standardised regimens of rehabilitation (usually physiotherapy and/or occupational therapy) soon after starting treatment to enable them to take advantage of the reduced spasticity. 
Table Al Construction of the Composite Function Index

Subjective assessments for each task (cleaning palms, cutting fingernails, putting arm through sleeve)

Items from Barthel ADL Index (arm section, items 3, 5, and 8)

\begin{tabular}{|c|c|c|c|c|c|c|c|c|}
\hline & Original & Adjusted & Feeding & & Dressing & & Grooming & \\
\hline Cannot do this activity & 4 & 0 & Unable & 0 & Unable & 0 & Unable & 0 \\
\hline A great deal of difficulty & 3 & 1 & Needs some help & 1 & Needs some help & 1 & Independent & 1 \\
\hline Moderate difficulty & 2 & 2 & Independent & 2 & Independent & 2 & & \\
\hline A little difficulty & 1 & 3 & & & & & & \\
\hline No difficulty & 0 & 4 & & & & & & \\
\hline
\end{tabular}

When these factors are taken into account, a clear link between reduced spasticity and increased function following botulinum toxin treatment is established.

\section{ACKNOWLEDGEMENTS}

We thank Professor Bakheit and all other investigators involved in the two published studies for all their work in collecting the original data.

\section{CONTRIBUTIONS}

The original idea came from HF, expanded later by DW and LT-S. All authors contributed to the development of the final paper. HF is the guarantor.

\section{Authors' affiliations}

H P Francis, R S Kingswell, C S Dott, E A Coxon, Ipsen, 190 Bath Road, Slough, SL1 3XE, UK

D T Wade, Oxford Chair for Enablement, Windmill Road, Oxford, OX3 7LD, UK

L Turner-Stokes, Herbert Dunhill Chair of Rehabilitation, King's College, London, UK

Competing interests: Ipsen produces and sells Dysport, a botulinum toxin type $A$ and has an interest in showing treatment benefit. All authors have a financial relationship with Ipsen

\section{APPENDIX A}

Construction of the Composite Function Index is shown in table AI.

\section{REFERENCES}

1 Bakheit AM, Thilmann AF, Ward AB, et al. A randomized, double-blind, placebo-controlled, dose-ranging study to compare the efficacy and safety of three doses of botulinum toxin type A (Dysport) with placebo in upper limb spasticity after stroke. Stroke 2000;31:2402-6.

2 Bakheit AM, Pittock S, Moore AP, et al. A randomized, double-blind, placebo-controlled study of the efficacy and safety of botulinum toxin type $A$ in upper limb spasticity in patients with stroke. Eur J Neurol 2001;8:559-65.

3 Bhakta BB, Cozens JA, Chamberlain MA, et al. Impact of botulinum toxin type A on disability and carer burden due to arm spasticity after stroke: a randomised double blind placebo controlled trial. J Neurol Neurosurg Psychiatry 2000:69:217-21.

4 Richardson D, Sheean G, Werring D, et al. Evaluating the role of botulinum toxin in the management of focal hypertonia in adults. J Neurol Neurosurg Psychiatry 2000;69:499-506.

5 Rodriquez AA, McGinn M Chappell R. Botulinum toxin injection of spastic finger flexors in hemiplegic patients. Am J Phys Med Rehabil 2000;79:44-7.

6 Simpson DM, Alexander DN, O'Brien CF, et al. Botulinum toxin type A in the treatment of upper extremity spasticity: a randomised, double-blind, placebocontrolled trial. Neurology 1996;46:1306-10.

7 Yablon SA, Agana BT, Ivanhoe $C B$, et al. Botulinum toxin in severe upper extremity spasticity among patients with traumatic brain injury: an openlabelled trial. Neurology 1996;47:939-44.

8 Bohannon RW, Smith MB. Inter-rater reliability of a modified Ashworth scale of muscle spasticity. Phys Ther 1987:67:206-7.

9 Brashear A, Gordon MF, Elovic E, et al. Intramuscular injection of botulinum toxin for the treatment of wrist and finger spasticity after a stroke. N Engl J Med 2002;347:395-400

10 Bhakta BB, Cozens JA, Bamford JM, et al. Use of botulinum toxin in stroke patients with severe upper limb spasticity. J Neurol Neurosurg Psychiatry 1996:61:30-5

11 Sheean GL. Botulinum treatment of spasticity: why is it so difficult to show a functional benefit? Curr Opin Neurol 2001;14:771-6.

12 Snow BJ, Tsui JKC, Bhatt MH, et al. Treatment of spasticity with botulinum toxin: a double blind study. Ann Neurol 1990;28:512-5.

13 Burbaud P, Wiart L, Dubos JL, et al. A randomised, double blind, placebo controlled trial of botulinum toxin in the treatment of spastic foot in hemiparetic patients. J Neurol Neurosurg Psychiatry 1996;61:265-9.

14 Smith SJ, Ellis E, White S, et al. A double-blind placebo-controlled study of botulinum toxin in upper limb spasticity after stroke or head injury. Clin Rehabil 2000;14:5-13.

15 Ada L, Canning C, Dwyer T. Effect of muscle length on strength and dexterity after stroke. Clin Rehabil 2000;14:55-61.

16 Canning CG, Ada L, O'Dwyer N. Slowness to develop force contributes to weakness after stroke. Arch Phys Med Rehabil 1999:80:66-70.

17 Lagalla G, Danni M, Reiter F, et al. Post-stroke spasticity management with repeated botulinum toxin injections in the upper limb. Am J Phys Med Rehabil 2000;79:377-84

18 Collin C, Wade DT, Davis S, et al. The Barthel ADL Index; reliability study. Int Disabil Stud 1988;10:61-3.

19 Ottenbacher KJ, Cusick A. Goal attainment scaling as a method of clinical service evaluation. Am J Occup Ther 1990;44:519-25.

20 Richardson D. Evaluation of interventions in the management of spasticity: treatment goals and out-come measures. In: Sheean G, ed. Spasticity rehabilitation. London: Churchill Communications Europe Ltd, 1998:57-69.

21 Brin M. Dosing, administration, and a treatment algorithm for use of botulinum toxin A for adult-onset spasticity. Muscle Nerve 1997;6:S208-20.

22 World Health Organisation. The International Classification of Functioning, Disability and Health (ICF). Geneva, Switzerland: WHO, 2002. 